\title{
Signal Reconstruction by Synchrosqueezed Wavelet Transform
}

\author{
Minsu Park ${ }^{a}$, Hee-Seok Oh ${ }^{a}$, Donghoh Kim ${ }^{1, b}$ \\ ${ }^{a}$ Department of Statistics, Seoul National University, Korea \\ ${ }^{b}$ Department of Applied Mathematics, Sejong University, Korea
}

\begin{abstract}
This paper considers the problem of reconstructing an underlying signal from noisy data. This paper presents a reconstruction method based on synchrosqueezed wavelet transform recently developed for multiscale representation. Synchrosqueezed wavelet transform based on continuous wavelet transform is efficient to estimate the instantaneous frequency of each component that consist of a signal and to reconstruct components. However, an objective selection method for the optimal number of intrinsic mode type functions is required. The proposed method is obtained by coupling the synchrosqueezed wavelet transform with cross-validation scheme. Simulation studies and musical instrument sounds are used to compare the empirical performance of the proposed method with existing methods.
\end{abstract}

Keywords: Cross-validation, empirical mode decomposition, intrinsic mode type function, synchrosqueezed wavelet transform, wavelets.

\section{Introduction}

The decomposition approach of a signal into a sum of well separated components has been widely studied over the last two decades. A popular approach is empirical mode decomposition (EMD) as proposed by Huang et al. (1998). EMD provides a data driven approach to decompose a signal into intrinsic mode functions (IMFs) according to the local oscillation magnitude in the physical domain. EMD constructs upper and lower envelopes by interpolating the local maxima and minima of a signal, respectively. Their average, mean envelope, produces a lower frequency component than the original signal by bisecting a signal evenly. By subtracting the mean envelope from the signal, a component $h$ is obtained, which is the highest-frequency pattern embedded in a signal. This procedure is repeatedly applied to the component $h$ until the component $h$ is locally symmetric with respect to zero. The resultant signal is called IMF. Then the signal is decomposed into the first IMF and the residue signal. Through the same process to the residue signal, a signal is sequentially decomposed into the finite number of IMFs and a residual signal.

Despite the widespread use in various fields, EMD has some limitations regarding theoretical properties since it is defined in an empirical way. To overcome this problem, Daubechies et al. (2011) proposed an alternative method, termed synchrosqueezed wavelet transforms (SWT), derived from

\footnotetext{
This work was supported by a grant from the Next-Generation BioGreen 21 Program (No.PJ008068), Rural Development Administration, Korea and the National Research Foundation of Korea (NRF) grant funded by the Korea government (MSIP) (2012R1A1A2039968 and 2012002717).

${ }^{1}$ Corresponding author: Department of Applied Mathematics, Sejong University, Gwangjin-Gu, Seoul 143-747, Korea.

E-mail: donghohkim@sejong.edu
}

Published 31 March 2015 / journal homepage: http://csam.or.kr

(c) 2015 The Korean Statistical Society, and Korean International Statistical Society. All rights reserved. 
reassignment methods of wavelet coefficients. Some studies based on SWT have been rapidly developed as follows: Thakur et al. (2013) discussed how various parameters are chosen in the discrete version and provided stability properties of synchrosqueezing with Gaussian white noise. Thakur and $\mathrm{Wu}$ (2011) and Meignen et al. (2012) dealt with the recovery process of a signal with non-uniform samples and noise via synchrosqueezing techniques. An extension of SWT by utilizing the wavelet packet transform for two-dimensional mode decomposition was proposed by Yang and Ying (2013).

However, the problem of reconstructing the underlying signal from noisy data has not been carefully studied yet. In this study, we propose a method to select some proper intrinsic mode components of SWT by a cross-validation approach. It can be expected that the proposed method provides a successful signal reconstruction based on SWT when the signal is contaminated by noise.

The remainder of this paper is organized as follows. Section 2 presents some background of SWT. In Section 3, the component selection method based on cross-validation is proposed. Section 4 provides some numerical studies to evaluate the empirical performance. Finally, concluding remarks are given in Section 5 .

\section{Background: Synchrosqueezed Wavelet Transforms}

The synchrosqueezing technique is effective to analyze and represent superposed signals in the timefrequency plane. The wavelet transform is also well organized to analyze mathematically and is widely recognized as effective tools for the signal analysis. Daubechies et al. (2011) employed the merits of these methods and introduced synchrosqueezing based on wavelets.

To make this paper self-contained, in this section, we summarize the concepts and theory of SWT from Daubechies et al. (2011). A signal $f(t)$ is defined as

$$
f(t)=\sum_{k=1}^{K} f_{k}(t),
$$

where each component $f_{k}(t)=A_{k}(t) \cos \left(\phi_{k}(t)\right)$ is an amplitude modulated-frequency modulated (AMFM) signal with amplitude $A_{k}(t)$ and positive frequency $\phi_{k}^{\prime}(t)$. Here, we use "prime" to denote the derivative with respect to time.

Definition 1. (Intrinsic mode type function) A continuous function $f: \mathbb{R} \rightarrow \mathbb{C}, f \in L^{\infty}(\mathbb{R})$ is said to be intrinsic-mode-type (IMT) with accuracy $\epsilon>0$ if $f(t)=A(t) e^{i \phi(t)}$ with $A$ and $\phi$ having the following properties:

$$
\begin{aligned}
& A \in C^{1}(\mathbb{R}) \cap L^{\infty}(\mathbb{R}), \quad \phi \in C^{2}(\mathbb{R}), \quad \phi^{\prime}, \phi^{\prime \prime} \in L^{\infty}(\mathbb{R}), \\
& \inf _{t \in \mathbb{R}} \phi^{\prime}(t)>0 \text { and } \sup _{t \in \mathbb{R}} \phi^{\prime}(t)<\infty, \\
& \left|A^{\prime}(t)\right| \leq \epsilon\left|\phi^{\prime}(t)\right| \text { and }\left|\phi^{\prime \prime}(t)\right| \leq \epsilon\left|\phi^{\prime}(t)\right|, \quad \forall t \in \mathbb{R}, \\
& M^{\prime \prime}:=\sup _{t \in \mathbb{R}}\left|\phi^{\prime \prime}(t)\right|<\infty
\end{aligned}
$$

Definition 2. (Superposition of IMT functions) The space $\mathcal{A}_{\epsilon, d}$ of superpositions of IMTs, with accuracy $\epsilon>0$ and separation $d$, consists of functions $f$ having the form

$$
f(t)=\sum_{k=1}^{K} f_{k}(t)=\sum_{k=1}^{K} A_{k}(t) e^{i \phi_{k}(t)},
$$


where $K>0$ and all the $f_{k}$ are IMT such that the $\phi_{k}$ satisfy

$$
\begin{aligned}
\phi_{k}^{\prime}(t) & >\phi_{k-1}^{\prime}(t), \\
\left|\phi_{k}^{\prime}(t)-\phi_{k-1}^{\prime}(t)\right| & \geq d\left[\phi_{k}^{\prime}(t)+\phi_{k-1}^{\prime}(t)\right], \quad \forall t \in \mathbb{R} .
\end{aligned}
$$

Any function in the class $\mathcal{A}_{\epsilon, d}$ is composed of several oscillatory components with slowly timevarying amplitudes, and any two adjacent functions are separated by at least $d$. The class $\mathcal{A}_{\epsilon, d}$ is a subset of $L^{\infty}(\mathbb{R})$ and details about this set is stated in Thakur and $\mathrm{Wu}(2011)$.

To analyze non-stationary signals, there are many time-frequency transforms such as continuous wavelet transform (CWT; Flandrin, 1999), short-time Fourier transform (STFT; Allen and Rabiner, 1977) and the Wigner-Ville distribution (WVD; Claasen and Mecklenbrauker, 1980). Synchrosqueezing is a special case of time-frequency (TF) reassignment that makes the spectrogram's energy more sharp around their center frequencies (Auger and Flandrin, 1995; Flandrin, 1999). Synchrosqueezing with the reconstruction process has been developed as an alternative approach for EMD, and its applications are considered (Daubechies et al., 2011; Thakur and Wu, 2011; Thakur et al., 2013). To understand the concept of SWT, we start with the continuous wavelet transform $W_{f}$ of the signal $f$ at scale $a$ and time shift $b$ that is defined as

$$
W_{f}(a, b)=a^{-\frac{1}{2}} \int f(t) \overline{\left(\frac{t-b}{a}\right)} d t,
$$

where $\psi$ is a mother wavelet using only the positive frequency.

Let's consider the simple form of a signal $f(t)=A \cos (\omega t)$ to illustrate the benefits obtained from SWT. By Plancherel's theorem and the Fourier transform of a signal $f(t)=A \cos (\omega t)$, the equation (2.1) can be written as

$$
W_{f}(a, b)=\frac{A}{4 \pi} a^{\frac{1}{2}} \overline{\hat{\psi}(a \omega)} e^{i b \omega},
$$

where $\hat{\psi}$ is a wavelet concentrated on the positive frequency axis. Then, a candidate instantaneous frequency $(\mathrm{IF}) \omega_{f}(a, b)$ is obtained from the CWT of $(2.2)$ as, for any $(a, b)$,

$$
\omega_{f}(a, b)=-i\left(W_{f}(a, b)\right)^{-1} \frac{\partial}{\partial b} W_{f}(a, b) .
$$

To reconstruct components and extract IFs, the information on the time-scale plane is moved to that of the time-frequency plane, that is, $(b, a) \rightarrow\left(b, \omega_{f}(a, b)\right)$. If the Fourier transform of $f$ is supported in $(0, \infty)$, then using following equation

$$
\int_{0}^{\infty} W_{f}(a, b) a^{-\frac{3}{2}} d a=\int_{0}^{\infty} \overline{\hat{\psi}(\xi)} \xi^{-1} d \xi \cdot \frac{1}{2 \pi} \int_{0}^{\infty} \hat{f}(\zeta) e^{i b \zeta} d \zeta
$$

the inverse CWT can be expressed as

$$
f(b)=\mathfrak{R}\left[\frac{1}{\mathcal{R}_{\psi}} \int_{0}^{\infty} W_{f}(a, b) a^{-\frac{3}{2}} d a\right],
$$

where $\mathcal{R}_{\psi}=(1 / 2) \int_{0}^{\infty} \overline{\hat{\psi}(\xi)} \xi^{-1} d \xi$. The curve on the synchrosqueezed wavelet transform is more sharply concentrated than that on the continuous wavelet transform. Figure 1 illustrates this fact for a test signal $f(t)=\cos (2 \pi t)$. 


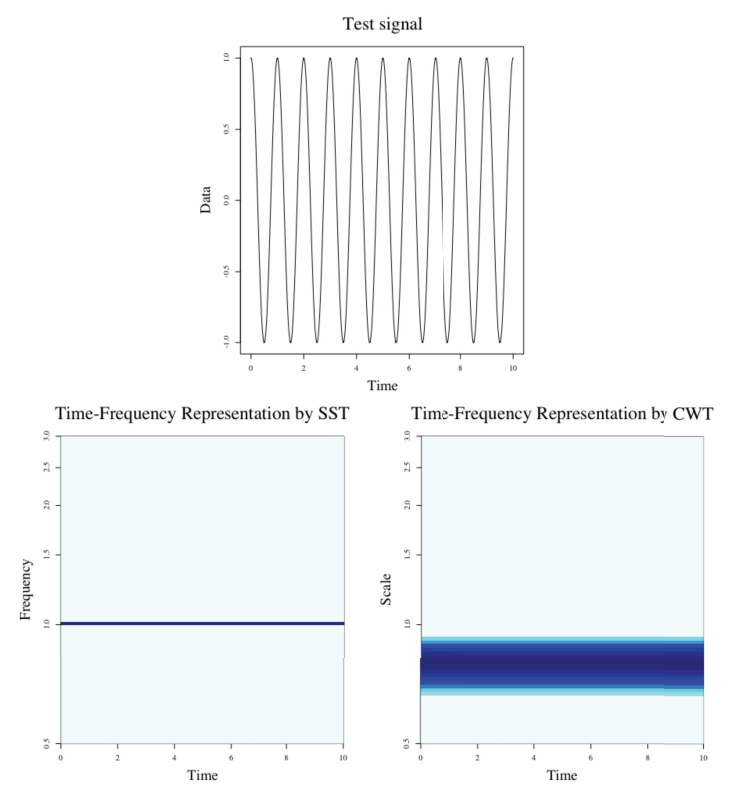

Figure 1: Top: A test signal $f(t)=\cos (2 \pi t)$; Bottom-left: The synchrosqueezed wavelet transform of $f$; Bottomright: The continuous wavelet transform of $f$.

Through the following theorem (Daubechies et al., 2011), we can see that the results obtained from synchrosqueezing are appropriate estimates for IFs and IMTs.

Theorem 1. (Daubechies et al., 2011) Let $f \in \mathcal{A}_{\epsilon, d}$ and $\tilde{\epsilon}=\epsilon^{1 / 3}$. Pick a function $h \in C_{c}^{\infty}$ with $\int h(t) d t=1$, and pick a wavelet $\psi$ such that its Fourier transform $\hat{\psi}$ is supported in $[1-\Delta, 1+\Delta]$, for some $\Delta<d /(1+d)$. Consider the function $S_{f, \tilde{\epsilon}}^{\delta}(b, \omega)$ obtained by synchrosqueezing $W_{f}$, with threshold $\tilde{\epsilon}$ and accuracy $\delta$, i.e.

$$
S_{f, \tilde{\epsilon}}^{\delta}(b, \omega):=\int_{A_{\tilde{\epsilon}, f}(b)} W_{f}(a, b) \frac{1}{\delta} h\left(\frac{\omega-\omega_{f}(a, b)}{\delta}\right) a^{-\frac{3}{2}} d a,
$$

where $A_{\tilde{\epsilon}, f}(b):=\left\{a \in \mathbb{R}_{+} ;\left|W_{f}(a, b)\right|>\tilde{\epsilon}\right\}$. Then, provided $\epsilon$ is sufficiently small, the following conditions hold for each $k$ :

- $\left|W_{f}(a, b)\right|>\tilde{\epsilon}$ only when, for some $k \in\{1, \ldots, K\},(a, b) \in Z_{k}:=\left\{(a, b) ;\left|a \phi_{k}^{\prime}(b)-1\right|<\Delta\right\}$, where $Z_{k}$ is defined as "scale band".

- For each $k \in\{1, \ldots, K\}$, and for each pair $(a, b) \in Z_{k}$ for which holds $\left|W_{f}(a, b)\right|>\tilde{\epsilon}$, we have

$$
\left|\omega_{f}(a, b)-\phi_{k}^{\prime}(b)\right| \leq \tilde{\epsilon} .
$$

- Moreover, for each $k \in\{1, \ldots, K\}$, there exists a constant $C$ such that, for any $b \in \mathbb{R}$,

$$
\left|\lim _{\delta \rightarrow 0}\left(\mathcal{R}_{\psi}^{-1} \int_{\left\{\omega:\left|\omega-\phi_{k}^{\prime}(b)\right|<\tilde{\epsilon}\right\}} S_{f, \tilde{\epsilon}}^{\delta}(b, \omega) d \omega\right)-A_{k}(b) e^{i \phi_{k}(b)}\right| \leq C \tilde{\epsilon},
$$

where $\mathcal{R}_{\psi}=\sqrt{2 \pi} \int \hat{\psi}(\zeta) \zeta^{-1} d \zeta$. 
This Theorem shows that the synchrosqueezed wavelet transform $S_{f, \tilde{\epsilon}}^{\delta}(b, \omega)$ is concentrated in narrow bands around instantaneous curves, and the wavelet synchrosqueezing transform can identify IF for each component and extract each component $f_{k}$ from the original signal $f$ using the inversion of the continuous wavelet transform at the narrow frequency bands. Estimated IFs can be extracted by existing ridge extraction approaches with high precision. A ridge is a continuous curve in the time-frequency plane, represented as a sequence of local amplitude peak. However, the problem of how many ridges are required is not easy; consequently, this study provides a suitable approach to this problem.

\section{Proposed Method}

\subsection{Cross-validation with SWT}

Cross-validation (CV) is used as a model validation technique in various areas including statistics. This method is useful to overcome the problem of overfitting, and it tends not to consider the whole data set but the partial data set when establishing a model. CV has two typical types: leave-one-out $\mathrm{CV}$ and $K$-fold CV. However, it is difficult to apply conventional CVs to the wavelet transform and SWT because Mallat's fast wavelet transform (Mallat, 1999) requires the number of data points to be dyadic and equally spaced.

Nason (1996) proposed the leave-half-out CV based on wavelets. He also provided the leave-oneout $\mathrm{CV}$ which is the modified version of the two-fold CV method; however, this algorithm suffers from boundary problems. Among imputation methods, the EM algorithm (Dempster et al., 1977) is popular to estimate incomplete data, and some approaches based on the EM algorithm for wavelets have developed by Lee and Meng (2005). Kim et al. (2006) proposed an imputation algorithm based on the $h$-likelihood, which provides a fast algorithm with good empirical performance. Consider leave-one-out CV. Given observations $\left\{t_{i}, y_{i}\right\}_{i=1}^{n}$, the remaining data except the $i^{\text {th }}$ observation are used as a training set. Since $t_{i}$ indicates location information and we usually deal with equally-spaced samples, we consider only these points $\left(y_{1}, \ldots, y_{i-1}, y_{i+1}, \ldots, y_{n}\right)$ as a training set, and $y_{i}$ as a test set. The number of the data set should be an integer power of 2 to employ the Mallat's fast algorithm, so that some methods must be considered for imputation of the removed $i^{\text {th }}$ observation. In this paper, we employ the iterative Min-Max imputation algorithm of Oh et al. (2009) for CV to determine how many significant components we need in the SWT representation. See Oh et al. (2009) for detailed imputation process. After this imputation process, we have the new datasets $\left(y_{1}, \ldots, y_{i-1}, y_{i}^{\prime}\right.$, $\left.y_{i+1}, \ldots, y_{n}\right)$, where $y_{i}^{\prime}$ is the imputed value, and wavelets can be applied.

This algorithm can be easily applied to any other CV methods. For 4-fold CV, as an example, the data set is divided into 4 roughly equal parts. Each fold is a test data set and the other 3 parts are training data sets. We regard each fold as missing values and impute the missing values. Figure 2 represents the imputed results using the above iterative Min-Max procedure for one fold from a noisy signal $y_{i}=f\left(t_{i}\right)+e_{i}, i=1, \ldots, 1024$, corrupted by Gaussian error $e$, where $f=\cos (2 \pi t)+\cos (3 \pi t)+$ $\cos (6 \pi t+0.75 \sin (2 / 5 \pi t)), t \in[0.5,3.0]$. Figure 2 shows that the iterative process produces efficient and effective result.

\subsection{Frequency curve selection on SWT}

As discussed in Section 2, the appropriate selection for the number of IF curves has not been investigated in Daubechies et al. (2011). It is easy to extract frequencies when the curves on the time-frequency (TF) plane are obvious to identify. However, when a signal is contaminated by noise, the frequencies having the energy spread out from the low frequency to the high frequency on the TF 

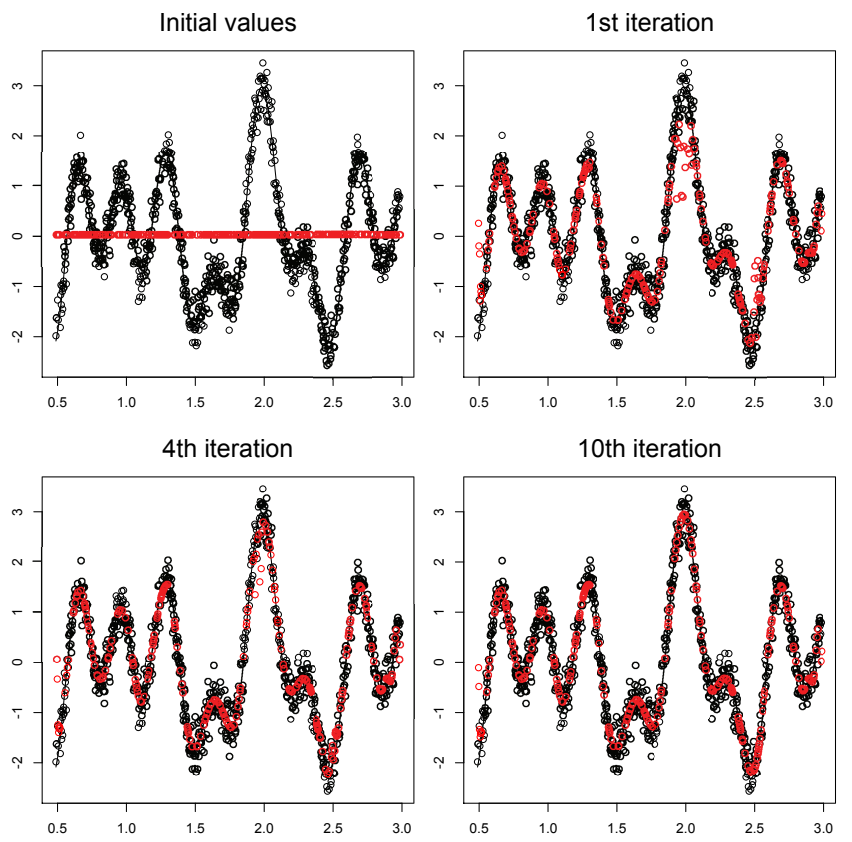

Figure 2: The example for the process of imputation. The black points represent 1,024 $\times(3 / 4)$ observations corrupted by Gaussian noise where the black line is the true signal. The initial values for $1,024 \times(1 / 4)$ missing points are mean of observed data. The red points are imputed values at iteration 1, 4 and 10.

plane; hence, it is difficult to determine how many frequency curves are needed. Thus, we provide an objective way to choose significant frequencies based on a combination of $\mathrm{CV}$ and imputation.

Suppose that signal $f_{N}$ consists of $N$ IMT functions that are corresponding to frequency curves. Given the noisy data $y_{i}$ 's observed from the model $y_{i}=f_{N}\left(t_{i}\right)+e_{i}$, we want to reconstruct $f_{N}$ by identifying significant frequency curves obtained from SWT. Note that the number $N$ of frequency curves is a parameter and an optimal $N$ will be chosen by the grid search for CV.

Given $N$ frequency curves, to employ $K$-fold $\mathrm{CV}$, the data set is divided into $K$ roughly equal parts. The $k^{\text {th }}$ fold $(k=1, \ldots, K)$ is a test set evaluated by the model based on the training set of the remaining $K-1$ parts. To perform SWT to the training set, we regard each $k^{\text {th }}$ fold as missing values and perform the imputation procedure explained in Section 3.1. Using the results of SWT for the training set and the imputed values in the $k^{\text {th }}$ fold, as described in Section 2, we obtain $N$ frequency curves by the existing ridge extraction method and reconstruct the signal for each $k^{\text {th }}$ fold. Let $\hat{f}_{N}^{-k}$ be the reconstructed signal for the $k^{\text {th }}$ fold. To validate the estimated model, we compute the predictive squared error $E_{k}$ for each $k^{\text {th }}$ fold, that is,

$$
E_{k}(N)=\sum_{i \in k^{t h} \text { fold }}\left(y_{i}-\hat{f}_{N}^{-k}\left(t_{i}\right)\right)^{2} .
$$

This provides the following cross-validation error,

$$
\mathrm{CV}(N)=\sum_{k=1}^{K} E_{k}(N)
$$


This process can be summarized as follows.

1. For a given $N$, calculate $\mathrm{CV}(N)$. For each $k=1,2, \ldots, K$,

1.1 Impute the values for each test set $y_{i}, i \in k^{\text {th }}$ fold.

1.2 Perform SWT, and extract $N$ frequency curves.

1.3 Obtain the reconstructed signal $\hat{f}_{N}^{-k}\left(t_{i}\right)$ based on $N$ frequency curves.

1.4 Calculate $\mathrm{CV}(N)$.

2. Find an optimal number $\hat{N}$ by minimizing $\mathrm{CV}(N)$.

3. Reconstruct $f_{N}$ by the estimated $\hat{N}$ frequency curves.

Unlike the conventional CV, the estimated model for each fold is employed with the whole dataset that consists of the training set and the imputed values for the test set. The data have always maintained to be an integer power of 2 because an imputation step is required before the estimation procedure. Thus, we apply this method to any types of $\mathrm{CV}$ to calculate the predictive error.

\section{Practical Performance}

In this section, we evaluate the effectiveness of the proposed method for the reconstruction problem based on SWT throughout several numerical examples. For the implementation, the R packages for SWT and the imputation approach using wavelets, SynchWave and CVThresh, are used.

\subsection{Simulated examples}

This section investigates the empirical performances of some methods including the proposed method. The three multiscale methods are considered as:

1. EMDThresh: the estimate of the underlying signal obtained by removing the highest frequency component decomposed from EMD.

2. WaveThresh: the estimate of the underlying signal obtained by SureShrink shrinkage of Donoho and Johnstone (1995).

3. SWTCv: the estimate of the underlying signal obtained by the proposed method.

As test signals, we consider the following two functions: $f_{1}(t)=\cos (2 \pi t)+\cos (3 \pi t)+\cos (6 \pi t+$ $0.75 \sin (2 / 5 \pi t))$ and $f_{2}(t)=\cos (\pi t)+\cos (2 \pi t)+\exp (-0.3 t) \cos (10 \pi t)+0.4 t$, where $t \in[0,10]$. Figure 3 shows the test signals $f_{1}, f_{2}$ and their components.

Figure 4 shows the time-frequency representation by SWT for noisy signal from test function $f_{1}$, the selection result of frequency curves and three recovered IMT functions by the proposed method based on 4-fold CV. Figure 5 depicts CV curves for 10 simulations according to the number of $N$. The proposed $\mathrm{CV}$ approach produces the minimum $\mathrm{CV}$ values at $N=3$. Figure 6 shows the reconstructed results according to different $N$ s for a test function $f_{1}$. The proposed method effectively produces the reconstructed signal with the choice $N=3$. Figure 7 shows the reconstruction results by other methods and the proposed method for noisy data from test function $f_{1}$. The figures indicates that the proposed method works better for signal reconstruction than other methods.

The following simulation study is conducted for further evaluation. To generate noisy data, we add Gaussian noise with a certain level of signal-to-noise ratio (SNR) into the true signal $f$, where 

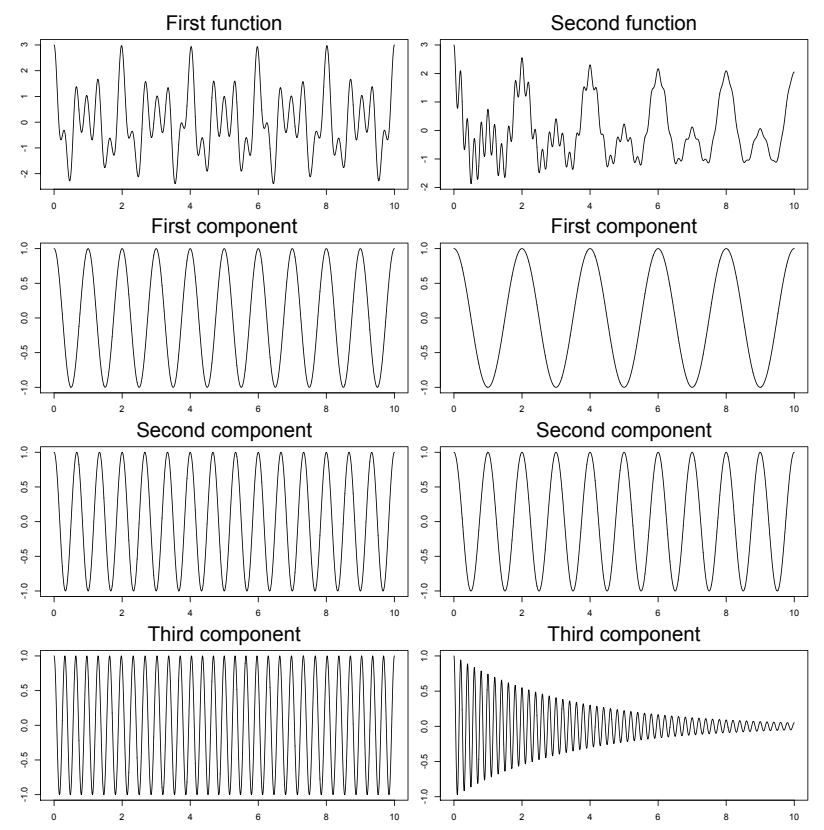

Figure 3: The test signals $\left(f_{1}\right.$ and $\left.f_{2}\right)$ and their components
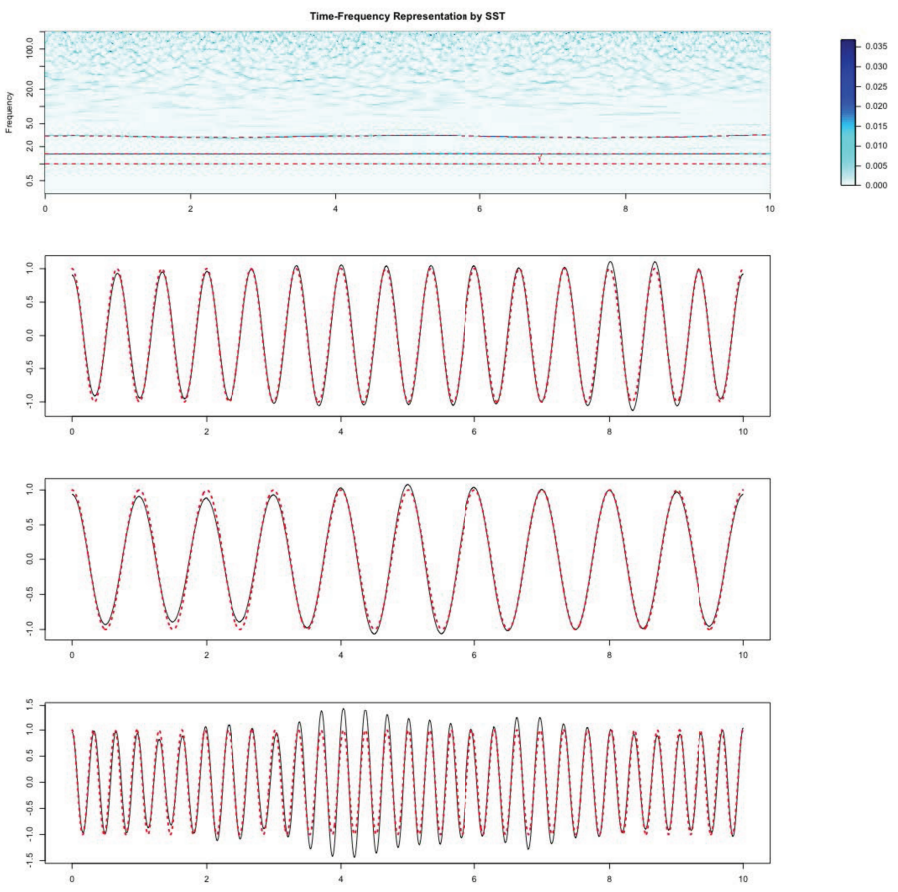

Figure 4: SWT for noisy signal from test function $f_{1}$ with three identified ridges (top) and the recovered IMT components with true signals denoted by red dots (next three panels). 


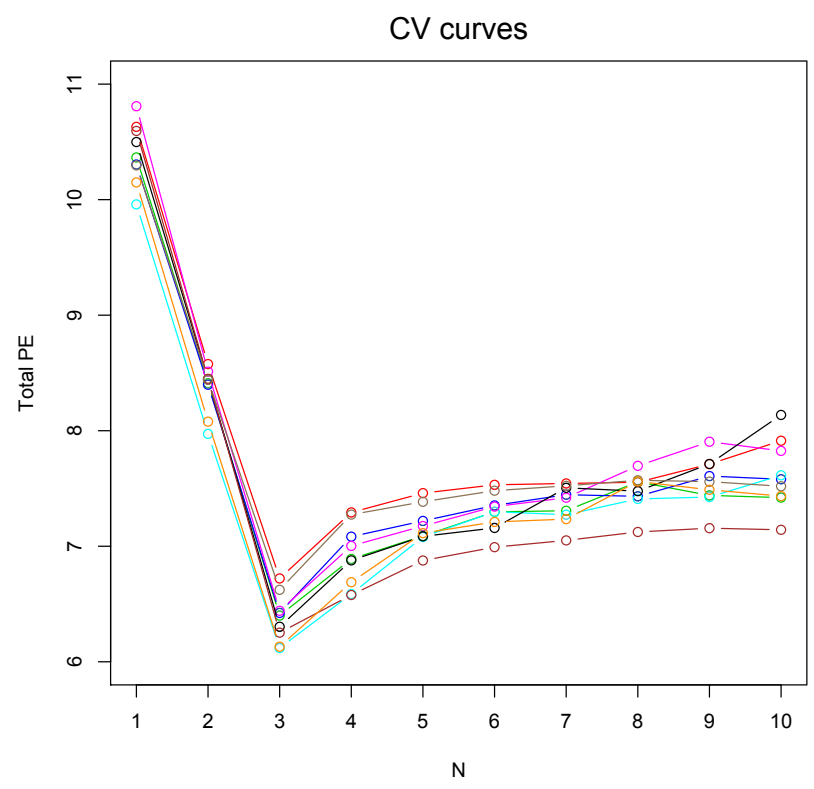

Figure 5: $C V$ values according to $N$ frequency curves for 10 simulation sets of noisy signal from test function $f_{1}$.

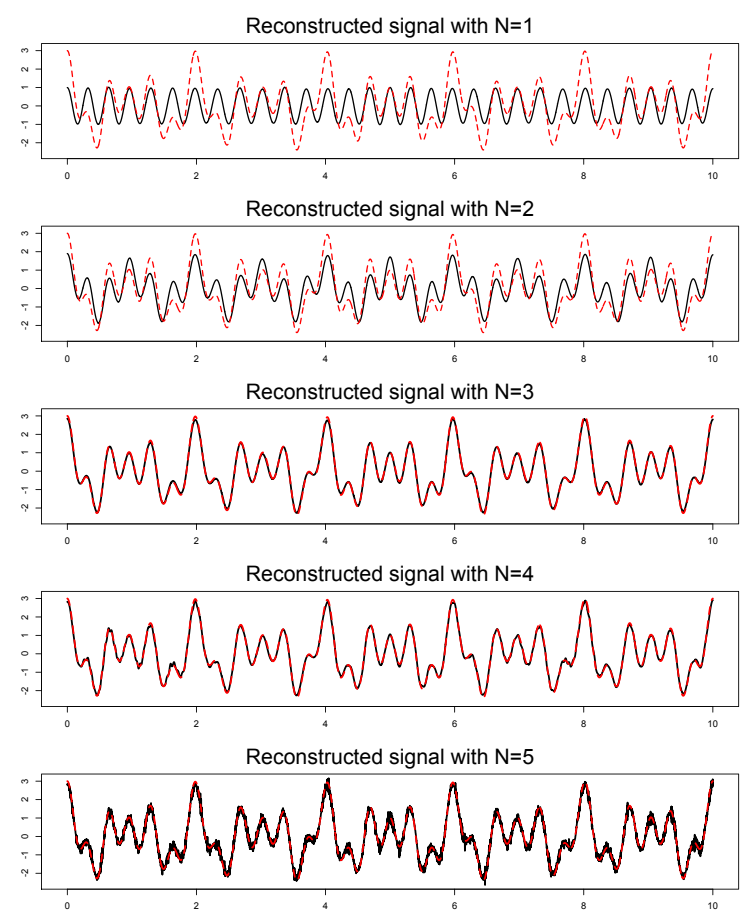

Figure 6: Reconstructed signals with different Ns. The red line represents the true signal, and black lines denote reconstructed signals. 

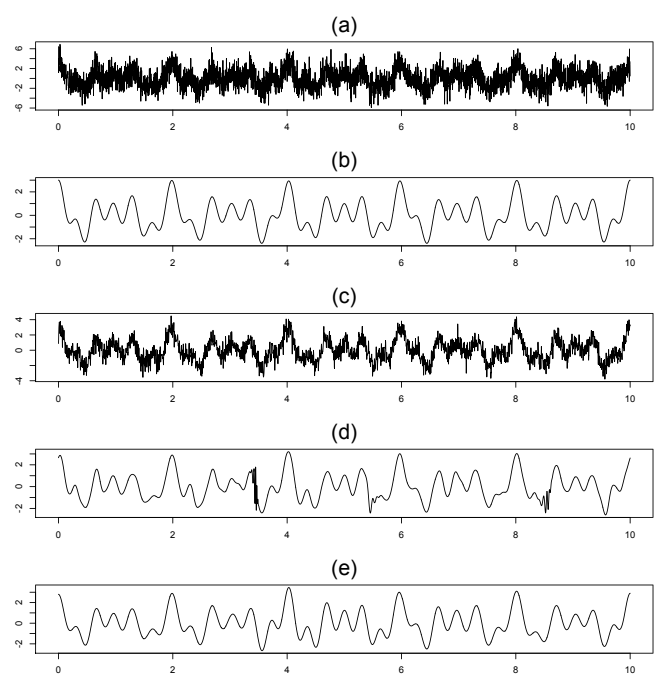

Figure 7: (a) Noisy signal from test function $f_{1}$, (b) true signal $f_{1}$, (c) reconstruction by EMDThresh, (d) reconstruction by WaveThresh, and (e) reconstruction by SWTcv.
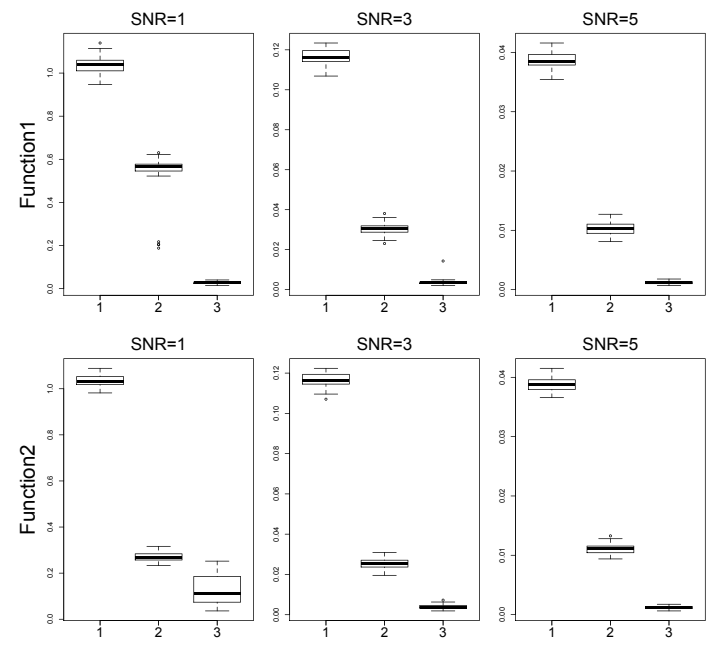

Figure 8: Boxplots of MSE values by (1) EMDThresh, (2) WaveThresh and (3) SWTcv.

SNR is defined as SNR $=\|f\| / \sigma$ where $\sigma$ is standard deviation of noise. We consider three levels of SNRs, 1,3, and 5. For each combination of the test function and SNR, 50 datasets were generated with a sample size of 4096. For each generated dataset, the three methods mentioned are applied to obtain an estimate of the test function $f$. As a measure of performance, the mean squared error, $\operatorname{MSE}\left(=\sum_{i=1}^{n}\left(f_{i}-\hat{f}_{i}\right)^{2} / n\right)$ is then calculated for each method. Figure 8 shows boxplots of MSE values by EMDThresh, WaveThresh and SWTcv. The proposed method outperforms two existing methods EMDThresh and WaveThresh for recovering the true function. 

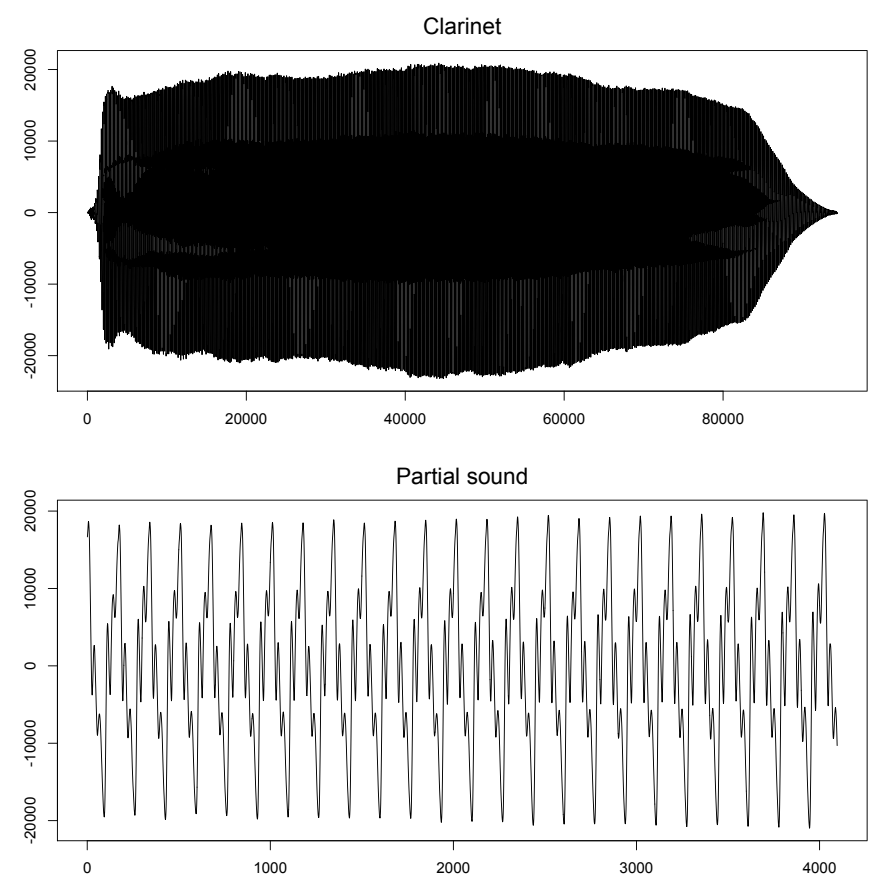

Figure 9: Musical instrument sound data of a clarinet (top) and partial parts of a clarinet (bottom).

\subsection{Real data analysis}

We perform the real data analysis for a signal produced by a musical instrument clarinet. The signal (http://wiki.laptop.org/go/Sound_samples) is produced by a clarinet playing the note Coctave0. The sound data are recorded at 44,100 samples per second with 16 bit resolution. The duration of a signal is 2.14 seconds. We extracted 4,096 samples between 0.32 to 0.41 seconds for computational cost (Figure 9).

To extract proper ridges, the proposed method is applied to the resultant energy of SWT. The number of ridges that minimizes $\mathrm{CV}$ by the proposed method is four. See Figure 10 of $\mathrm{CV}$ values according the number $N$.

Figure 11 shows the identified ridges based on the proposed method from time-frequency representation; consequently, four recovered IMT components are represented in Figure 12. The results indicate that the signal produced by clarinet contains several periodic waves induced by blowing a stream of air over the embouchure hole. In order to evaluate the reconstruction ability, the results of the proposed method are compared with the original sound signal. Figure 13 describes the original sound and the reconstructed sound as the sum of four frequency curves obtained by the proposed method. Figure 13 indicates that the reconstructed sound effectively represents the original sound. The proposed method identifies appropriate ridges in the representation of SWT, and extracts several periodic waves, which cannot be captured by visual inspection.

\section{Concluding Remarks}

In this study, a reconstruction method of a signal is proposed by the optimal selection for the number of frequency curves (or IFs) on the time-frequency plane. In the literature, the process of choosing a 


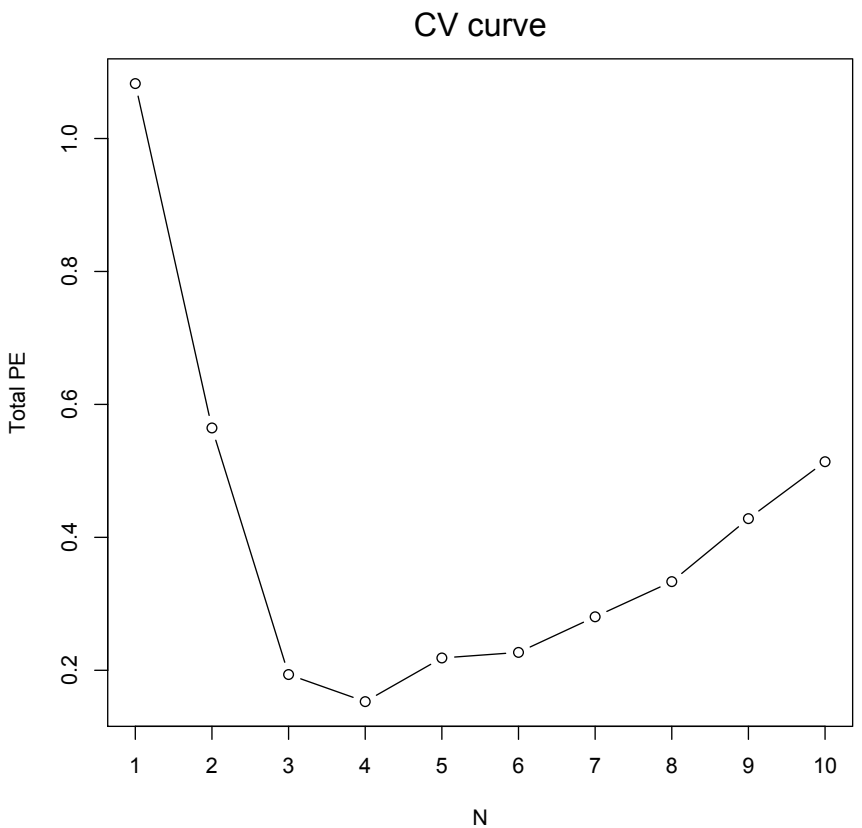

Figure 10: $C V$ values for $N$ frequency curves based on 4-fold $c v$.

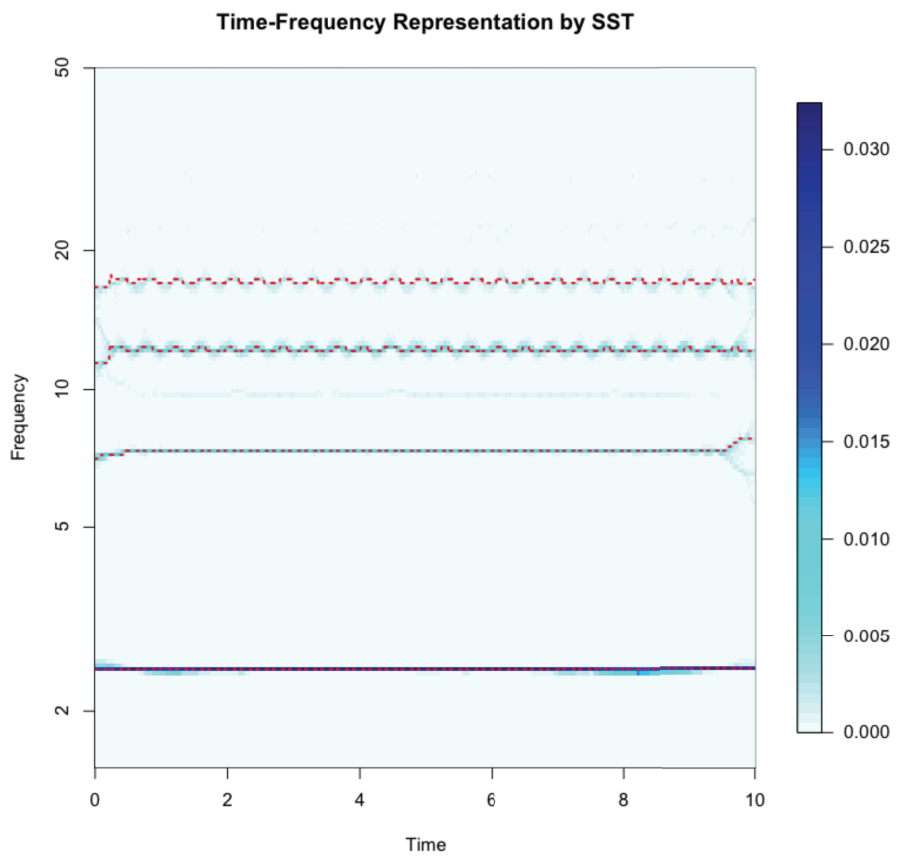

Figure 11: Time-frequency representation of SWT and identified ridges (red dots). 

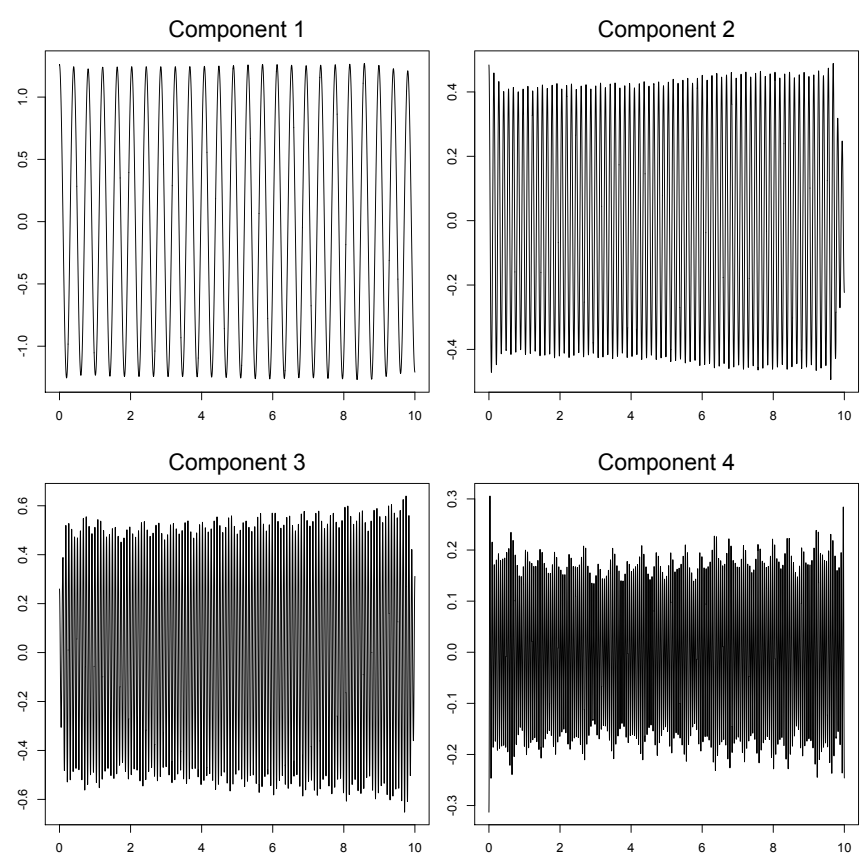

Figure 12: Four recovered IMT components using the proposed method from the clarinet sound.

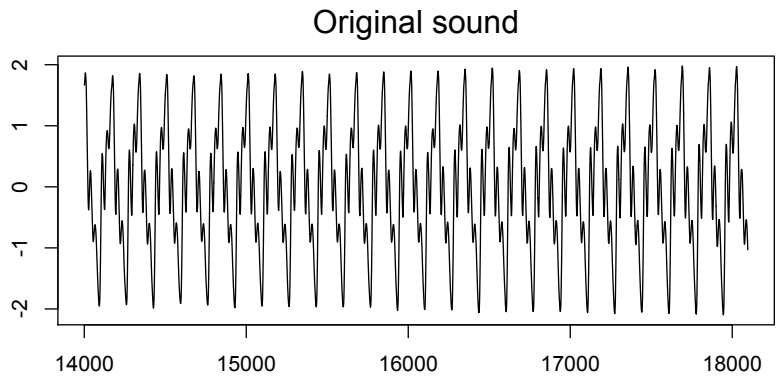

Reconstructed sound

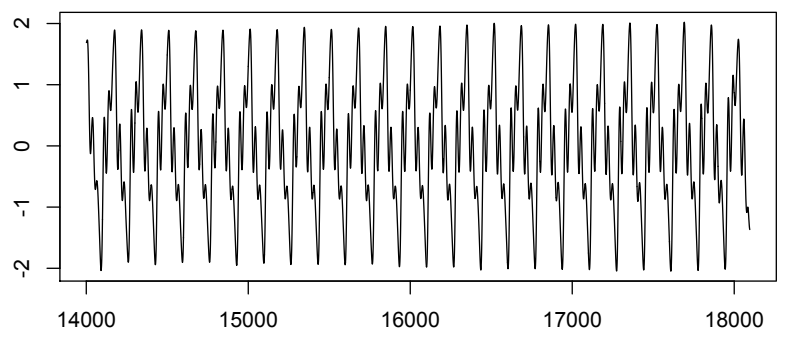

Figure 13: The original clarinet signal and reconstructed sound. 
candidate of IF feature is very ad-hoc. The proposed method is based on a cross-validation technique for assessing the result. The signal reconstruction by the proposed method is easily applicable since the usage of $\mathrm{CV}$ for wavelets is available through the imputation technique. The simulation studies show its effectiveness and efficiency.

\section{References}

Allen, J. B. and Rabiner, L. R. (1977). A unified approach to short-time Fourier analysis and synthesis, Proceedings of the IEEE, 65, 1558-1564.

Auger, F. and Flandrin, P. (1995). Improving the readability of time-frequency and time-scale representations by the reassignment method, IEEE Transactions on Signal Processing, 43, 1068-1089.

Claasen, T. and Mecklenbrauker, W. F. C. (1980). The Wigner distribution: A tool for time frequency signal analysis, Philips Journal of Research, 35, 217-250.

Daubechies, I., Lu, J. and Wu, H. T. (2011). Synchrosqueezed wavelet transforms: An empirical mode decomposition-like tool, Applied and Computational Harmonic Analysis, 30, 243-261.

Dempster, A. P., Laird, N. M. and Rubin, D. B. (1977). Maximum likelihood from incomplete data via the EM algorithm, Journal of the Royal Statistical Society: Series B, 39, 1-38.

Donoho, D. L. and Johnstone, I. M. (1995). Adapting to unknown smoothing via wavelet shrinkage, Journal of the American Statistical Association, 90, 1200-1224.

Flandrin, P. (1999). Time-Frequency/Time-Scale Analysis, Academic Press.

Huang, N. E., Shen, Z., Long, S. R., Wu, M. L., Shih, H. H., Zheng, Q., Yen, N. C., Tung, C. C. and Liu, H. H. (1998). The empirical mode decomposition and Hilbert spectrum for nonlinear and nonstationary time series analysis, Proceedings of the Royal Society of London A, 454, 903-995.

Kim, D., Lee, Y. and Oh, H.-S. (2006). Hierarchical-likelihood-based wavelet method for denoting signals with missing data, IEEE Signal Processing Letters, 13, 361-364.

Lee, T. C. M. and Meng, X. L. (2005). A self-consistent wavelet method for denoising images with missing pixels, In Proceedings of the 30th IEEE International Conference on Acoustics, Speech, and Signal Processing, 2, 41-44.

Mallat, S. (1999). A Wavelet Tour of Signal Processing, Academic Press.

Meignen, S., Oberlin, T. and McLaughlin, S. (2012). A new algorithm for multicomponent signals analysis based on synchrosqueezing: With an application to signal sampling and denoising, IEEE Transactions on Signal Processing, 60, 5787-5798.

Nason, G. P. (1996). Wavelet shrinkage by cross-validation, Journal of the Royal Statistical Society: Series $B, \mathbf{5 8}, 463-479$.

Oh, H.-S., Kim, D. and Lee, Y. (2009). Cross-validated wavelet shrinkage, Computational Statistics, 24, 497-512.

Thakur, G., Brevdo, E., Fuckar, N. S. and Wu, H.-T. (2013). The synchrosqueezing algorithm for time-varying spectral analysis: Robustness properties and new paleoclimate applications, Signal Processing, 93, 1079-1094.

Thakur, G. and Wu, H.-T. (2011). Synchrosqueezing-based recovery of instantaneous frequency from nonuniform samples, SIAM Journal on Mathematical Analysis, 43, 2078-2095.

Yang, H. and Ying, L. (2013). Synchrosqueezed wave packet transform for 2D mode decomposition, SIAM Journal on Imaging Sciences, 6, 1979-2009. 